\section{Volkskrankheit Arteriosklerose}

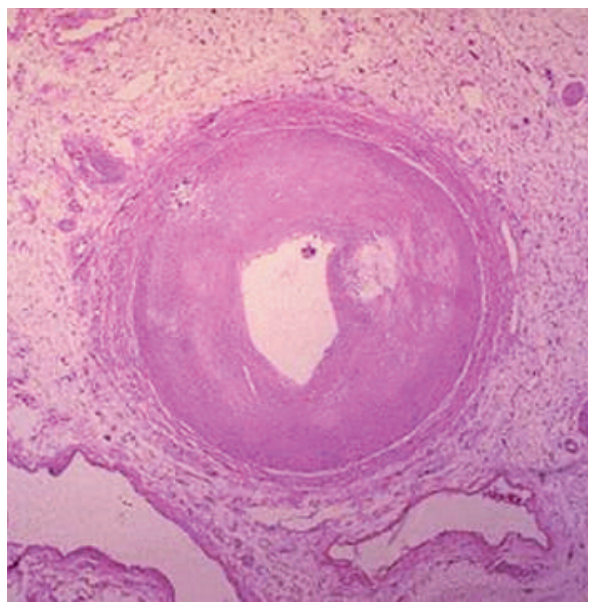

Eine neue Studie stellt die zentrale Rolle des

Botenstoffs Interleukin-1beta bei Arteriosklerose in Frage.

\section{Licht in Sicht}

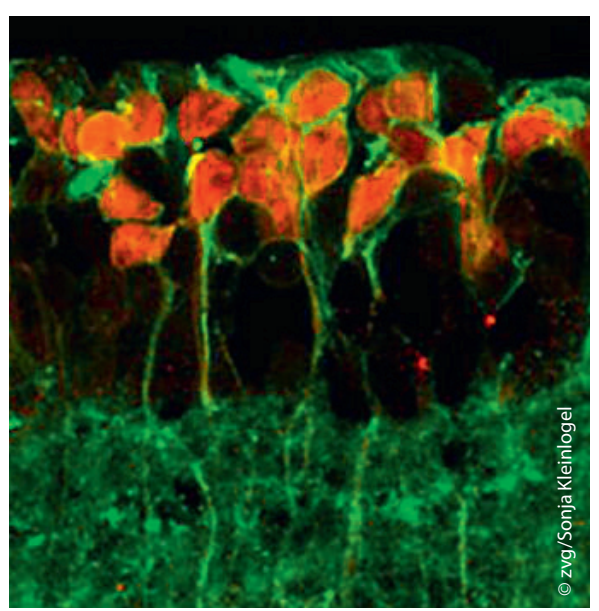

Netzhaut einer blinden Maus, die mit OptomGluR6 (rot markiert) therapiert wurde.

Un engagement pour une respiration libérée Déclenché par des protéines - de pollen de graminées, d'épithélium du chat ou d'acariens notamment - I'asthme allergique touche 7 à 15\% des enfants et 6 à $7 \%$ des adultes en Suisse. aha! Centre d'Allergie Suisse offre aux personnes concernées des informations complètes, des conseils et des formations. L'application «Info asthme» permet de vérifier si l'asthme est bien contrôlé et propose aussi des prévisions météorologiques, des informations sur les polluants atmosphériques et sur les charges polliniques en français, en allemand et en italien.

(aha! Centre d'Allergie Suisse)
Herz-Kreislauf-Erkrankungen sind die Todesursache Nummer eins in der Schweiz. Häufig liegt eine Arteriosklerose zugrunde. In einem Forschungsprojekt an der ETH Zürich hat nun Dr. Stefan Freigang einen wichtigen Auslöser der Arteriosklerose entdeckt. Die gefährlichen Ablagerungen bei einer Arteriosklerose sind die Folge einer chronischen Entzündung der Blutgefässe, ausgelöst durch zu viel LDLCholesterin. Immunzellen nehmen das Cholesterin auf, woraufhin ein Rezeptor den Botenstoff Interleukin-lbeta aktiviert und so eine Entzündungsreaktion auslöst. Stefan Freigang stellt nun die zentrale Rolle des Botenstoffs Interleukin-1beta in Frage. "Unsere Studie deckt einen völlig neuen Weg der Entzündungsreaktion in den Gefässen auf», so der Forscher. Massgeblich beteiligt ist eine zweite Variante des Botenstoffs, das Interleukin-1alpha.

(Schweizerische Herzstiftung)

Weltweit leiden etwa zwei Millionen Menschen an der vererbbaren und unheilbaren Augenkrankheit Retinitis pigmentosa. Nach und nach verlieren die Betroffenen ihr Augenlicht, da die Fotorezeptoren absterben. «Auch wenn die Fotorezeptoren ihre Funktion verlieren, sind die darunter liegenden Nervenzellen noch voll funktionsfähig», erläutert Sonja Kleinlogel vom Institut für Physiologie der Universität Bern. «Diese verbleibenden Zellen kann man lichtempfindlich machen und sozusagen zu Ersatzfotorezeptoren umwandeln.» Die Forschenden haben Mäuse, die von Geburt an unter Retinitis pigmentosa leiden, mit Hilfe dieses Ansatzes therapiert. Das Ergebnis: Die therapierten Mäuse konnten wieder Tageslicht sehen, auf visuelle Reize reagieren und durch Licht ausgelöste Verhaltensmuster erlernen.

(Universität Bern)
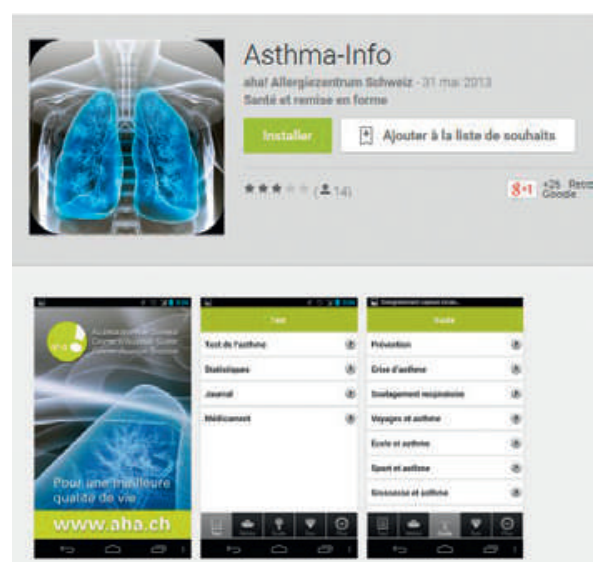

Fibres de coton

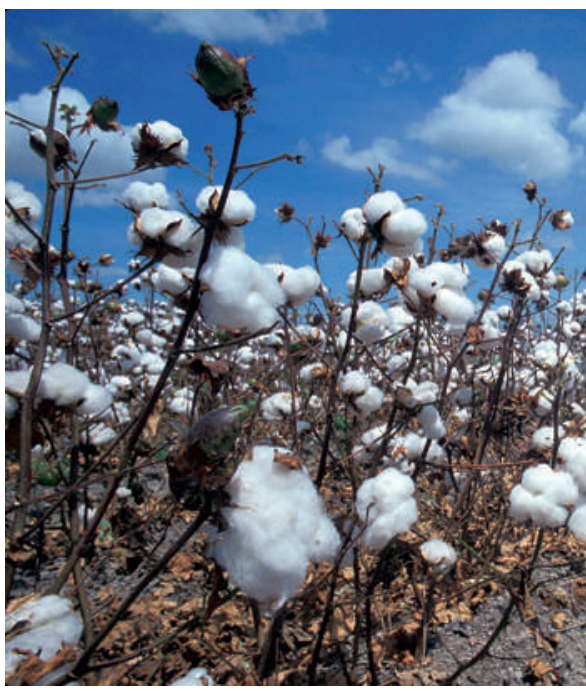

L'inhalation de nanofibres végétales peut-elle porter atteinte aux poumons?

Dans le cadre du Programme national de recherche "Opportunités et risques des nanomatériaux" (PNR 64), une équipe de chercheurs a étudié si l'inhalation de nanofibres végétales pouvait porter atteinte aux poumons. Les résultats montrent que les nanofibres végétales ne présentent pas de risque immédiat pour la santé. Toutefois, les chercheurs rapportent que le système de cellules pulmonaires parvient à éliminer efficacement les fibres courtes, alors que les fibres plus longues demeurent à la surface des cellules. On n'est donc pas en mesure de déterminer si les fibres longues peuvent avoir à long terme des effets délétères sur les poumons. Les expériences avec les nanotubes de carbone avaient déjà démontré que le contact des cellules pulmonaires avec de longs tubes perturbe leur équilibre, car elles tentent en vain de leur faire traverser la membrane cellulaire.

(FNS)

Unterstützend: Geschichten von Patientinnen und Angehörigen

Obwohl das Betroffensein an einer psychischen Erkrankung häufig ist, fühlen sich Betroffene und deren Angehörige trotzdem oft alleine, einsam und unverstanden. Was hilft, sind Erzählungen über das persönlich Erlebte. Anhand dieser konkret erlebten Erfahrungen über den Umgang mit der Erkrankung, aber auch das Berichten über den Weg in die Genesung, ermutigt und fördert einen gemeinsamen Erfahrungsaustausch. Um diese Patienten- und Genesungsgeschichten zu sammeln, gibt es eine empfehlenswerte Internetseite - www.patientengeschichten.ch -, die gegen die Einsamkeit helfen soll.

(Pro mente sana) 